\title{
Planning the trajectory of manipulator gripper with a parallel structure and flexible links
}

\author{
Vadim Dubovskov ${ }^{1, *}$, Yuriy Valyukevich ${ }^{1}$ \\ ${ }^{1}$ Don State Technical University, 344000 Rostov-on-Don, Russia
}

\begin{abstract}
Traditionally, the tasks of the objects transportation in working space are solved using manipulation robots with a sequential kinematic structure. However, the increasing requirements for the mechanical rigidity, load-carrying capacity, mass-size and speed parameters caused the need to develop a new classes of manipulation robots with a parallel connecting circuit. The closed kinematic scheme of such manipulators provides a more higher rigidity of the entire structure. The reduced mass of moving parts reduces the load on electric drives and thus provides an increase in manipulator gripper dynamics and the accuracy of positioning. Finally, the energy efficiency of the process increases. The problems of planning the trajectory of manipulator gripper with a parallel structure and flexible links are considered within the paper.
\end{abstract}

\section{Parallel structure manipulator}

As the name implies, a cable-driven parallel manipulator, or cable robot, is a type of parallel manipulator in which the platform is driven by a set of cables in place of traditional rigid links. These cables are sometimes referred to as tendons, or wires. A winch, consisting of a tensioning motor and spool, is used to adjust the length or tension in each cable. Coordinated retraction and extension of the cables allows for the position and orientation of the platform to be controlled. Cable-driven systems offer many advantages over traditional parallel manipulators. Replacement of the heavy prismatic actuators with relatively light cables facilitates performance at higher accelerations and allows for potentially vast workspaces. Mounting of the winches to the fixed base further reduces the system's moving inertia. Compared to the moving platform, the cables have relatively low mass and inertial properties and thus, their effects are oftentimes considered negligible - an assumption which greatly simplifies the modeling and analysis. The simple design of cable-driven robots also gives rise to a system that is reconfigurable, less expensive to construct and maintain, and easily transported, disassembled, and re-assembled.

The limitations in parallel robot mechanism paved a path for the cable robots. The research was directed towards developing a mechanism that would keep the characteristics of parallel robot intact and reduce the drawbacks, especially small workspaces. The abilities of parallel robots to move with high speed and accelerations, stiff structure, high force carrying capacity were important to be kept intact.
Workspace computation is an important aspect of robot design, as it gives an idea of reachability of the robot.

The kind of workspace required depends largely on the application of the system. As discussed in [6] of the paper, there are many ways to analyse the workspace of a robot. The force feasible workspace approach is adopted for this study as it is based primarily on the force values. A feasible workspace is considered as a set of positions and orientations of a robot in which the EEs are controllable, tensions in cables are positive, forces values lie within the pre-set bounds and the EE is far from singularity.

Despite their numerous advantages, there are several challenges associated with cable-driven robots. The fact that cables can pull but not push gives rise to a unilateral constraint wherein the cables must always be maintained in tension. As a result, the modeling and analysis methods that have been developed for conventional rigid-link manipulators cannot be directly applied to cable-driven systems. Furthermore, this unilateral property gives rise to the need for actuation redundancy - that is, more cables than degrees of freedom (DoF) - in order to completely restrain and control the platform. In fact, if the number of DoF in the system is denoted by $m$, and the number of cables is denoted by $n$, it has been shown [9-10] that a fully-restrained system requires at least $n=m+1$ cables. This concept can be illustrated through a simple example [5]. Consider a rigid body in the horizontal plane that is subjected to linear motion, i.e. a system with one degree of freedom. We wish to control the motion of the rigid body using cables. If we place a cable on one side of the mass - say, to the left-hand side - it is obvious that the mass can easily be pulled to the left. However, in such a configuration, the mass cannot be moved to the right, as

\footnotetext{
* Corresponding author: dybovskov@mail.ru
} 
the cable cannot exert a compressive force. Any attempt to do so will result in the cable becoming slack. By adding one more cable on the opposite side of the rigid body, motion along both directions can now be controlled. This idea can be extended to systems with greater degrees of freedom.

\section{Schematic of the manipulator}

Schematic of the manipulator with flexible links is presented in fig. 1 .

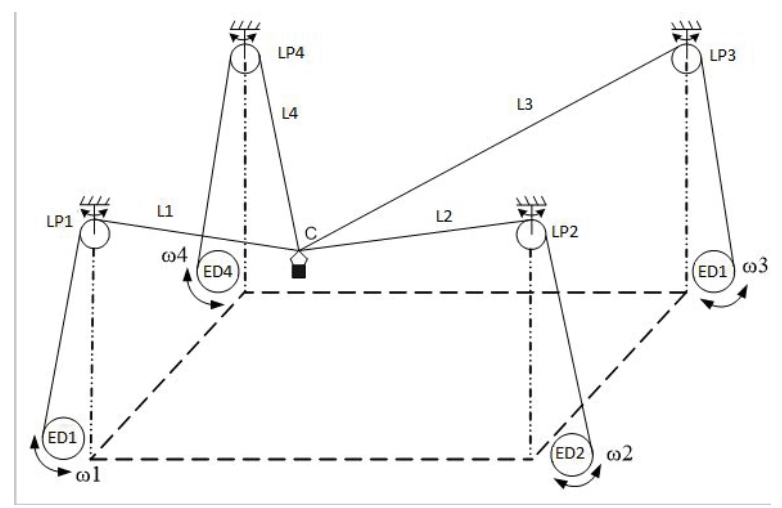

Fig. 1. Schematic of the manipulator with flexible links

The following notations are accepted for the fig. 1: ED1-ED4 - electric drives of the manipulator links № 14 respectively; LP1-LP4 - link pulleys № 1-4 respectively; L1-L4 - links (cables) № 1-4 respectively; $\mathrm{C}-$ the manipulator gripper; $\omega 1-\omega 4$ - angular velocities of the electric drives № 1-4 respectively; $\varphi 1-\varphi 4$ - angles of rotation of the link pulleys № 1-4 respectively.

The solution of direct and inverse kinematics of position and speed for the structure presented in fig. 1 is available in [1,2]. Based on it the two methods of trajectory planning are proposed. The first one have a significant methodological error in accuracy. The second one requires a high speed of the hardware and software complex that implements the manipulator control system. The task of developing the control actions on electric drives of links can be formulated as follows:

- the trajectory segment is given as a straight line segment (fig. 2);

- the absolute coordinates of gripper position and the generalized coordinates of all links at the beginning and end of the segment are known;

- the current values of generalized coordinates are could be measured at any point of motion trajectory;

- the speed of motion and the sampling frequency of control actions on speed of the electric drives links are given.

The method of planning the trajectory is based on the calculation of instantaneous velocity in the direction of each of generalized coordinates as a function of velocity vector oriented along the displacement trajectory in absolute coordinates at regular intervals. Fig. 2 shows an example of the relative position of vectors with a given velocity magnitude along the trajectory and the velocity vector of one of the generalized coordinates.

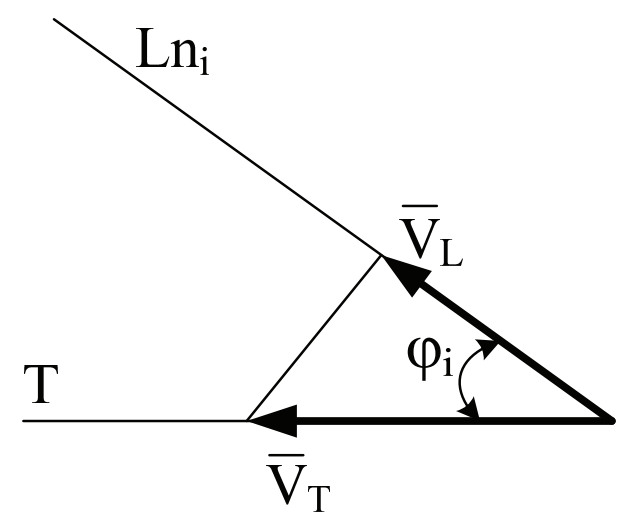

Fig. 2. Velocity vector diagram of the gripper motion along given trajectory

The following notations are accepted for the fig. 2: $\bar{V}_{T}$ - velocity vector of gripper motion along the trajectory; $\bar{V}_{L}$ - velocity vector of the manipulator link motion; $\varphi_{i}$ - angle between the vectors $\bar{V}_{T}$ and $\bar{V}_{L}$.

The module of instantaneous velocity for each manipulator link is determined from the relation:

$$
V_{L n i}=V_{T} \cos f_{n i}
$$

The index $n$ corresponds to the link number and the index $i$ - to the number of current iteration.

The angle $\varphi_{i}$ can be found by the cosine theorem from the triangle $\triangle O N i M$ (fig. 3), which is formed by a segment of trajectory $N i M$, initial (segment $O N i$ ) and final $(O M)$ positions of the manipulator link $\mathrm{n}$ (fig. 1).

$$
\cos \phi_{1 i}=\frac{L n_{N i}^{2}+L_{M N i}^{2}-L n_{M}^{2}}{2 L n_{N i} \cdot L_{N M i}}
$$

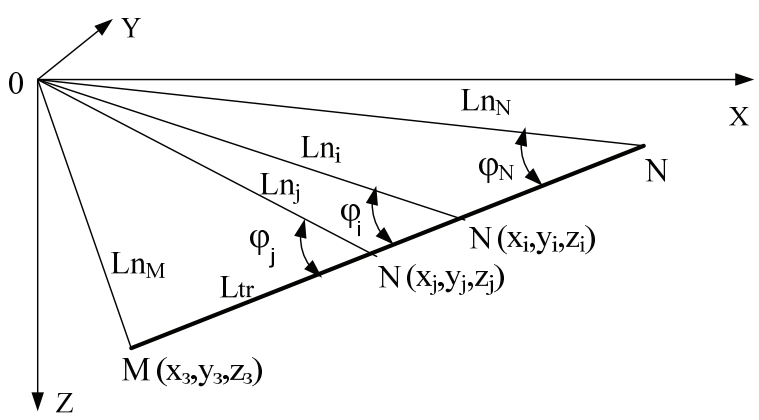

Fig. 3 - An example of the gripper motion trajectory in absolute coordinates $\mathrm{x}, \mathrm{y}, \mathrm{z}$

The final values of generalized (point $M$ of the trajectory segment) and Cartesian coordinates are known from the condition of problem. The current value of generalized coordinate (segment $O N$ of the triangle) is known from the position sensor data. The current values of Cartesian coordinates of point $\mathrm{Ni}$ can be determined by solving the direct problem of manipulator's kinematics, providing that the values of remaining generalized ones 
are known from condition of the problem. The current length of segment $N M i$ is common for all links and determined by the Cartesian coordinates of current and final points of trajectory in the manipulator working space.

The motion from one reference point to the other within a trajectory is performed with a fixed generalized velocity along each coordinate. Estimated number of the trajectory segment division, can be determined from the relation:

$$
K=\frac{L_{t r}}{V_{T} \cdot \tau}
$$

Where the Ltr - initial length of trajectory segment; $\tau$ - the time per iteration.

The denominator in Equation 3 corresponds to an elementary displacement $\Delta l$ during the time of one iteration $\tau$. The step per one iteration for each Cartesian coordinate is determined from the relation:

$$
\begin{gathered}
\Delta x=\frac{X_{N}-X_{M}}{K} ; \\
\Delta y=\frac{Y_{N}-Y_{M}}{K} ; \\
\Delta z=\frac{Z_{N}-X_{M}}{K}
\end{gathered}
$$

In equations 4 , the index $N$ corresponds to the initial point coordinates and the index $M$ - to the final point coordinates within the trajectory segment.

The error of proposed planning method can be divided into two components: a deviation error from desired trajectory and an absolute error due to difference between the instantaneous velocity vectors along the trajectory and the generalized coordinate, as shown in fig. 4.

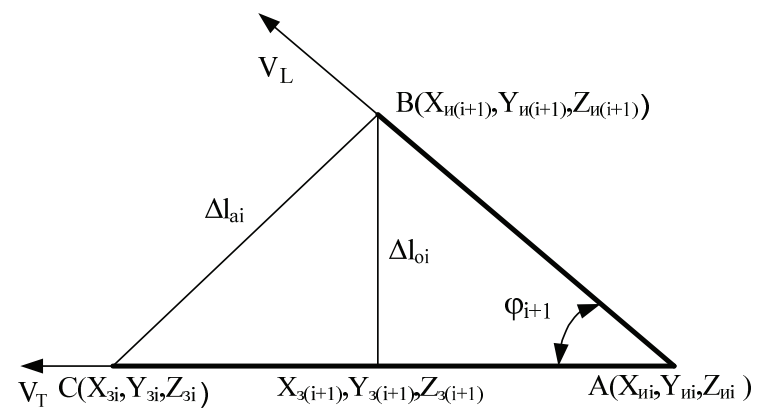

Fig. 4 - Methodological errors of the planning method

Estimating the accuracy of motion along desired trajectory the absolute error is not significant since it's corrected at each step due to true values of initial point coordinates used for each iteration - $X_{u(i+l)}, Y_{u(i+l)}, Z_{u(i+l)}$. The error magnitude is determined by misalignment of the true and desired velocity values along the trajectory. This is a specific property of proposed method which is not the scope of this paper.

The value of relative error $\Delta l o i$, providing that the $\triangle A B C$ vertices coordinates (fig. 4) are known can be determined quite simply by the methods of analytical geometry. It should be noted that the absolute coordinates $X_{u(i+1)}, Y_{u(i+1)}, Z_{u(i+1)}$ are determined based on the three generalized coordinates. Thus the current initial point coordinates within the segment are corrected relating to the ideal case presented in the fig. 2 at every step of motion along the trajectory. It is obvious from the equation 3 that the magnitude of relative error is depends on ratio between the motion velocity along trajectory $V_{T}$ and the sampling frequency $1 / \tau$ of control actions on the electric drive of manipulator link.

\section{Planning method tested}

The presented planning method was tested on the real model of manipulator performed in accordance with the kinematic scheme shown in fig. 1 . The working space of manipulator's layout is represented by a parallelepiped $2100 \times 1600 \times 2500 \mathrm{~mm}$. The control system is a hierarchical two-level one.

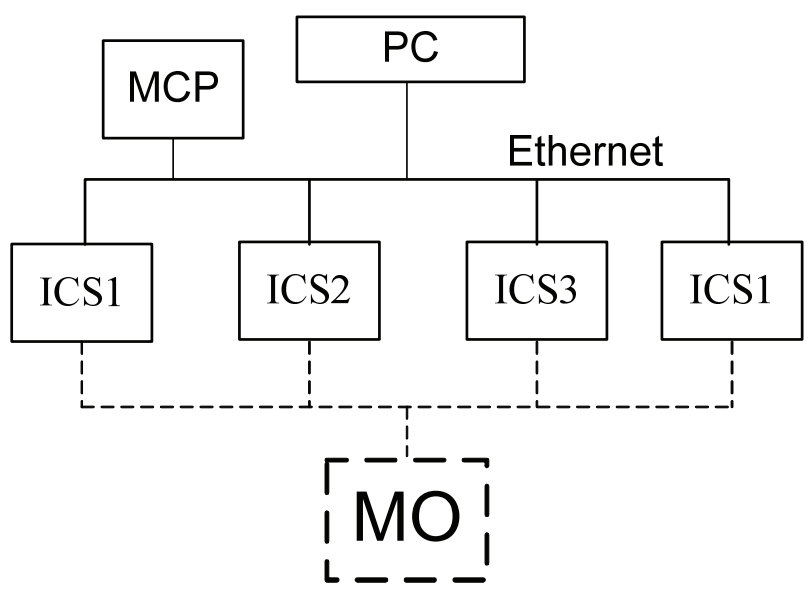

Fig. 5 - General functional scheme of manipulator control

The following notations are accepted for the figure 5: PC - personal computer; ICS1-ICS4 - informationcontrol systems of the manipulator links 1-4 respectively; MO - moving object; MCP - manual control panel.

It is obvious from the fig. 5 that the control system is a hierarchical two-level one. The first level is implemented on a personal computer in software way and solves the following range of control tasks:

-the initial initialization of the control system;

-collection and analysis of the state parameters of the control systems 3in1-3in4;

-performing the control actions on the electric drives ACS of the manipulator links;

-displaying state of the ACS parameters during the moving process.

The second level includes the ACS for each of the links and for the information support of control process of the upper hierarchy level. Communication between all devices is based on a wired link in the Ethernet standard, protocol UDP.

The upper control level problem have been solved using a program shell of information control, implemented in $\mathrm{C}++$ language under the operating system Windows 7 . 
Fig. 6 presents a screenshot of the manipulator control program user interface in the mode of moving by a given trajectory. The current gripper position in frontal and horizontal projection of working space is continuously displayed in the corresponding graphical windows of screen interface. The numerical values of parameters mentioned above are displayed in the corresponding tabular windows of screen interface. Each desired position of gripper is determined by position of the sliders. The desired trajectory is also displayed in graphical windows in the frontal and horizontal projections of working space. In the mode of motion, the true trajectory is superimposed on the desired one and the current values of Cartesian and generalized coordinates as well as the relative error are displayed in the corresponding cells of the tables.

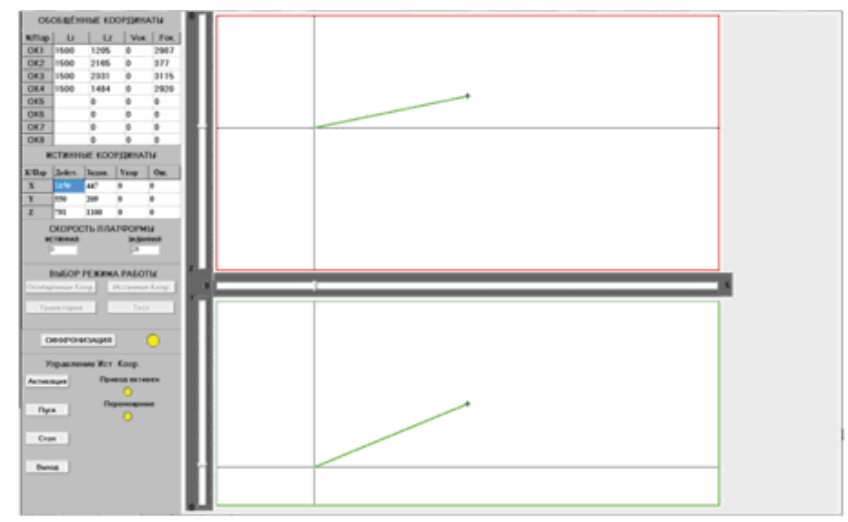

Fig. 6. Screenshot of the manipulator control interface window.

The trial operation showed a full efficiency of proposed method for planning the trajectory of moving manipulator gripper. Maximum deviation from a desired trajectory for the $V_{T}=200 \mathrm{~mm} / \mathrm{s}$ and $\tau=20 \mathrm{~ms}$ was $\Delta$ lomax $=5 \mathrm{~mm}$. For the $V_{T}=50 \mathrm{~mm} / \mathrm{s}$ and $\tau=20 \mathrm{~ms}$ the value of relative error was within plus or minus 4 discretes of measured generalized coordinates (one discrete is equal to $0.154 \mathrm{~mm}$ ).

\section{Conclusions}

In this paper, the methods of planning the trajectory of moving manipulator gripper was considered. The method based on the calculation of instantaneous velocity value for each of generalized coordinates and on the formation of control actions on ACS of the links electric drives. The magnitude of error and the factors affecting the method are determined. The results of full-scale modeling on the manipulator real model base are presented.

\section{References}

1. Yu.A. Valyukevich, A. V. Alepko, Technical science, no. 6 (2011) [in Russian]

2. Manikandan Bakthavatchalam, François Chaumette, and Eric Marchand, IEEE International Conference on Robotics and Automation, 5521- 5526 (2013)
3. Marco Carricato and Jean-Pierre Merlet, IEEE International Conference on Robotics and Automation, 3011-3017, (2011)

4. Jordan B., Batalin M., and Kaiser W., IEEE International Conference on Robotics an Automation, 144-150 (2017)

5. Lumia, Ronald, In proc. International Symposium on Robotics and Manufacturing, Maui, HI, (1994)

6. Riechel A., Bosscher P., Lipkin H., and Ebert-Uphoff I., In Proc. 10th International Topical Meeting on Robotics and Remote Systems for Hazardous Environments, (2004)

7. Shanmugasundram A.P. and Moon F.C., ASME International Mechanical Engineering Congress and Exposition, 57, 55-65 (1995)

8. Shiang W., Cannon D., and Gorman J., In Proc. 1999 IEEE International Conference on Robotics and Automation (1999)

9. Takemura F., Enomoto M., Tanaka T., Denou K., Kobayashi Y., and Tadokoro S., In Proc. 2005 IEEE/ASME International Conference on Advanced Intelligent Mechatronics, 658-663 (2005)

10. Takemura F., Maeda K., and Tadokoro S., In Proc. IEEE/RSJ International Conference on Intelligent Robots and Systems, 3504-3509 (2007)

11. Williams R. L., Gallina P., and Vadia J., Journal of Robotic Systems, no.3 (2003)

12. Williams R. L. and Gallina P., In Proc. 2001 ASME Design Technical Conference (2001) 